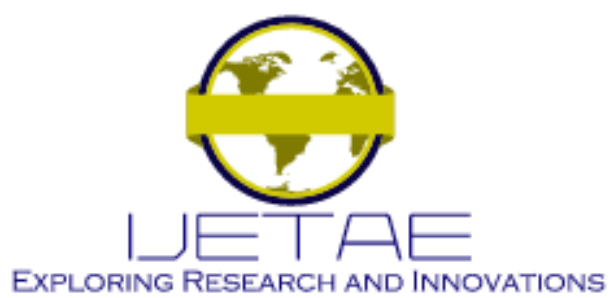

International Journal of Emerging Technology and Advanced Engineering

Website: www.ijetae.com (E-ISSN 2250-2459, Scopus Indexed, ISO 9001:2008 Certified Journal, Volume 11, Issue 10, October 2021)

Manuscript Received: 05 September 2021, Received in Revised form: 08 October 2021, Accepted: 15 October 2021

DOI: 10.46338/ijetae1021_02

\title{
Study of Goal Oriented Obstacle Avoidance for Mobile Robot
}

\author{
Yeon Taek $\mathrm{OH}$ \\ School of Mechanical Engineering, Tongmyung University, 428 Sinseon-ro, Nam-gu, Busan, Korea
}

\begin{abstract}
This paper suggests simultaneous mechanism which makes UGV to secure its safety and recreate optimum path right after obstacle avoidance occurs. Research target and sensors is shown and the suggested optimum region path algorithm is presented. Also, this paper provides the performance of suggested algorithm by simulation, and will propose the direction of the future plans. The fixed and varying weight algorithm satisfy the constraints, once the robot arrives at the goal position in the simulation. However, the fixed weight algorithm was not able to drive on the shortest path, which is optimal, with many other successful weight values. On the other hand, the varying weight algorithm successfully generated the optimized path by changing the weight values in term of local environment. Especially, in the second simulation, the robot was able to safely arrive to the goal with little time period. This paper proposed VTV and Local Optimal path algorithm that the robot can generate the optimal path to the goal position without colliding obstacles as adapting Fuzzy controller which continuously optimizes the weight values of the cost function in terms of local environment.
\end{abstract}

Keywords - obstacle avoidance, virtual force field, unmanned ground vehicle, optimal path design, weight control method

\section{Introduction}

In the future warfare, unmanned vehicles and robots will execute the battle and the battle support mission. It is predicted that the utilizability of the unmanned system will be larger within the hazardous places. unmanned systems are actively used in noncombatant sectors, such as collecting information, dangerous work, guard work and has proved its efficiency which makes possible to predict that unmanned system will be use wider. Especially, Unmanned ground vehicle(UGV) is regarded as a main stream of the future warfare which can be representative of 'Future Combat System'. Basically, UGV can be operated by the supervisor with radio communication. However, on the certain situation like war, the reliability cannot be always guaranteed. To operate its missions on the certain and limited situation, autonomous ability is essential and basically, it can be realized by the path planning.
Autonomous technology of robots can be categorized to 'Localization', 'Mapping' and 'Path planning'. Localization is the problem of using sensor measurements to estimate the robot's pose relative to some map. Localization must also deal with sensor noise and with uncertainty in the map. Mapping is the problem of collecting and correlating a multitude of sensor measurements into a common map representation. There are many possible map representations, the main distinction being between featurebased maps, which are lists of features and information about them, and featureless metric maps, which directly represent the geometry of the environment. Path planning is the problem of deciding what the robot should try to do next. Planning operates under constraints, such as safety, and it must also deal with uncertainty: in the map, in the current localization estimate, and in the outcome of actions. Usually, combined 'Localization' and 'Mapping' technology is called 'SLAM (Simultaneous Localization and Mapping). Above those three technologies, 'Mapping' is an important technology for robots to reach its local goal with using optimal path by avoiding obstacles. 'Path planning' for UGV includes mission plan, region path plan, local path plan, and emergency obstacle avoidance. When UGV has general information, it can create path autonomously and this is called 'Region path plan'. 'Local path plan' and 'Emergency obstacle avoidance' basically follows the 'Region path plan' but, can recreate path when unrespectable structures and obstacles are detected by UGV's sensors.

Reconnaissance UGV can provide useful intelligence by infiltrating into the battlefield based on their 'region path plan'. However, due to unexpected obstacles on region path, UGV should modify its 'local path plan' by using sensors.

Researches of 'region path plan' and 'emergency obstacle avoidance' algorithm have been continued. Virtual Force Field (VFF) uses the 'potential field method' [1]. Basic concept of this method is making virtual attraction force from local goal and virtual repulsion force from obstacles. VFF is simple and easy to realize, but also has demerits like zigzag movement on narrow path or local minima stuck matter. 


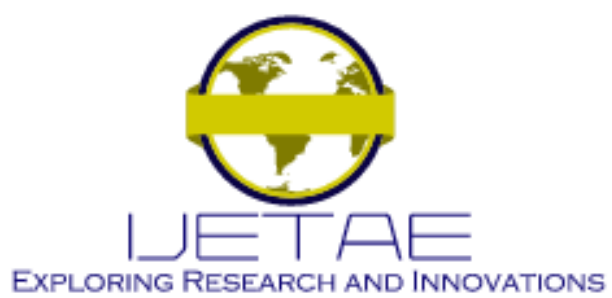

International Journal of Emerging Technology and Advanced Engineering

Website: www.ijetae.com (E-ISSN 2250-2459, Scopus Indexed, ISO 9001:2008 Certified Journal, Volume 11, Issue 10, October 2021)

Vector Field Histogram (VFH) is a method that can supplement VFF [2]. By creating radial form range around the robot, obstacle density on steering area can be schematize with polar histogram, so UGV can move to the direction which has the lowest obstacle density. VFH can make UGV's movement more stabilized, but have not solved the local minima problem on $\mathrm{U}$ shaped obstacle. Dynamic Window Approach (DWA) is a velocity-based obstacle avoidance method using robot dynamics [3]. DWA defines 'Dynamic Window', the set of possible operating velocities which are in velocity space with linear velocity axis and angular velocity axis. After that, algorithm extracts the best velocity from a cost function which includes path generation keeping current speed, safe distance from obstacle and local goal. As a result, DWA has its strength on excluding dynamical impossible path, but its weakness on sensitivity for the inner variables directly effects on the performance of algorithm. In the other hand, Nearness Diagram (ND) is a rule-based algorithm with 'Divide and conquer' concept [4]. It can manage the entire situation by four standards and five decisions. Due to its clear decisions, ND can make robots to have great performance even in the extremely small area. However, this method does not consider dynamics and kinematics of robots which makes hard to apply on robots accept omni-directional robots.

All the above concept of methods and developers are summarized on Table 1.

TABLE 1.

ALGORITHM COMPARISON

\begin{tabular}{|c|l|l|}
\hline Algorithm & \multicolumn{1}{|c|}{ Developer } & \multicolumn{1}{c|}{ Concept } \\
\hline $\begin{array}{c}\text { Virtual } \\
\text { Force } \\
\text { Field }\end{array}$ & $\begin{array}{l}\text { Johann Borenstein et } \\
\text { al., University of } \\
\text { Michigan, USA }\end{array}$ & $\begin{array}{l}\text { - Fusion of potential method and } \\
\text { histogram grid } \\
\text { Virtually attractive and } \\
\text { repulsive forces considered each } \\
\text { for a goal and obstacle }\end{array}$ \\
\hline $\begin{array}{c}\text { Vector } \\
\text { Field } \\
\text { Histogram }\end{array}$ & $\begin{array}{l}\text { Johann Borenstein et } \\
\text { al., Univ. of }\end{array}$ & $\begin{array}{l}\text { Extraction of candidate } \\
\text { direction based on polar } \\
\text { histogram generated from } \\
\text { peripheral sensor data }\end{array}$ \\
\hline $\begin{array}{c}\text { Dynamic } \\
\text { Window } \\
\text { Approach }\end{array}$ & $\begin{array}{l}\text { Dieter Fox et al., } \\
\text { Germany of Bonn, }\end{array}$ & $\begin{array}{l}\text { Path planning based on both } \\
\text { translational and rotational } \\
\text { velocity to consider dynamics } \\
\bullet \text { Choice of best velocities by } \\
\text { optimization of an objective } \\
\text { function }\end{array}$ \\
\hline $\begin{array}{c}\text { Nearness } \\
\text { Diagram }\end{array}$ & $\begin{array}{l}\text { Javier Minguez et al., } \\
\text { Univ. of Zaragoza, } \\
\text { Spain }\end{array}$ & $\begin{array}{l}\text { 'Divide and Conquer' strategy } \\
\text { Only 5 situations and actions } \\
\text { for 4 criterions }\end{array}$ \\
\hline
\end{tabular}

Before it is mentioned, 'region path plan' and 'emergency obstacle avoidance' has various application fields and has shown superior performance, but still it needs more research for region area based UGV's safety and optimum path creation. Previous researches and suggested algorithms are concentrated on obstacle avoidance and drive problem settlement. However, this research suggests simultaneous mechanism which makes UGV to secure its safety and recreate optimum path right after obstacle avoidance occurs. Research target and sensors will be shown and the suggested 'optimum region path algorithm' will be presented. Also, this paper will provide the performance of suggested algorithm by simulation.

\section{Virtual Tangential Vector Method (VTV)}

Complex area including multiple obstacles like woods and rocks has been selected as an UGV operation environment. Besides high-density obstacles, narrow passage has been also included in the environment due to its mission, passing narrow space. To make narrow passage to have the same condition as a complex area with obstacles, test environment has been settled as follows. Ackermann vehicle, which has front steering function, is selected to UGV. The size of UGV is defined as $2 \mathrm{~m}(\mathrm{~W}) \times$ $3 \mathrm{~m}(\mathrm{~L})$ on simulation and to assure the space for UGV's movement, passage width has been selected to be $5 \mathrm{~m}$, which is the half of the robot's diagonal length. Global positioning system(GPS), Inertia measurement unit(IMU), and Laser measurement system(LMS) is installed in this UGV. GPS is installed to measure the current location and local goal. IMU is for getting the posture information. LMS is installed to measure the distance and degree between the vehicle and obstacles. Performing simulations, we assumed LMS as $0.1 \mathrm{~m}$ resolution and maximum $20 \mathrm{~m}$ range to distance direction, and $0.5 \mathrm{~m}$ resolution and maximum $270^{\circ}$ scan to degree direction for easy calculations. Assumed those above mentioned sensors are all installed in the middle of the UGV.

\section{A. Encirclement and Enlargement of Obstacle}

There are three steps used in the proposed algorithm. The first is to model the obstacle as a circle by three points information. The second is to model the robot as a mass point; the encircled obstacle is enlarged to compensate this reduction. The third is to define a direction, along which the mobile robot has to move to get away from a trap. 


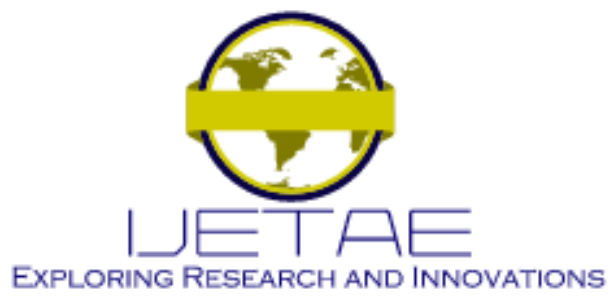

International Journal of Emerging Technology and Advanced Engineering

Website: www.ijetae.com (E-ISSN 2250-2459, Scopus Indexed, ISO 9001:2008 Certified Journal, Volume 11, Issue 10, October 2021)

When a mobile robot is moving in an unknown environment, the LMS obtains information of three points about obstacles: two end points, and a nearest point which is detected by the sensor. With the three points information, a unique circle is constructed on Fig. 1. The mobile robot is modelled as a mass point and the encircled obstacle is then enlarged to compensate for this reduction on Fig. 2. The resulting obstacle replacement by the encirclement and enlargement processes is referred to a virtual obstacle. Using the notations on Fig. 3, the detail geometric quantities and the moving direction are summarized as formulas in Eq. 1 below.

$$
\begin{aligned}
& P_{k, \text { ini }}=\left(x_{k, \text { ini }}, y_{k, \text { ini }}\right) \\
& P_{k, \text { end }}=\left(x_{k, \text { end }}, y_{k, \text { end }}\right) \\
& P_{k, \text { near }}=\left(x_{k, \text { near }}, y_{k, \text { near }}\right) \\
& x_{k, \text { ini }}=r_{k, \text { ini }} \times \cos \alpha_{k, \text { ini }}, y_{k, \text { ini }}=r_{k, \text { ini }} \times \sin \alpha_{k, \text { ini }} \\
& x_{k, \text { end }}=r_{k, \text { end }} \times \cos \alpha_{k, \text { end }}, y_{k, \text { end }}=r_{k, \text { end }} \times \sin \alpha_{k, \text { end }} \\
& x_{k, \text { near }}=r_{k, \text { near }} \times \cos \alpha_{k, \text { near }}, y_{k, \text { near }}=r_{k, \text { near }} \times \sin \alpha_{k, \text { near }} \\
& y_{a}=m_{a}\left(x-x_{k, \text { ini }}\right)+y_{k, \text { ini }} \\
& y_{a}=m_{a}\left(x-x_{k, \text { near }}\right)+y_{k, \text { near }} \\
& m_{a}=\frac{y_{k, \text { near }}-y_{k, \text { ini }}}{x_{k, \text { near }}-x_{k, \text { ini }}}, \quad m_{b}=\frac{y_{k, \text { end }}-y_{k, \text { near }}}{x_{k, \text { end }}-x_{k, \text { near }}} \\
& y^{\prime}{ }_{a}=-\frac{1}{m_{a}}\left(x-\frac{x_{k, \text { ini }}+x_{k, \text { near }}}{2}\right)+\frac{y_{k, \text { ini }}+y_{k, \text { near }}}{2} \\
& y_{b}^{\prime}=-\frac{1}{m b_{a}}\left(x-\frac{x_{k, n e a r}+x_{k, \text { end }}}{2}\right)+\frac{y_{k, \text { near }}+y_{k, \text { end }}}{2} \\
& x_{k}^{a b s}=\frac{m_{a} m_{b}\left(y_{k, \text { ini }}-y_{k, \text { end }}\right)+m_{b}\left(x_{k, \text { ini }}-x_{k, \text { near }}\right)-m_{a}\left(x_{k, \text { near }}-x_{k, \text { end }}\right)}{2\left(m_{a}-m_{b}\right)} \\
& y_{k}^{a b s}=\left.y_{a}^{\prime}\right|_{x_{k}^{a b s}} \\
& R_{\text {cir }}=\sqrt{\left(x_{k, n e a r}-x_{k}^{a b s}\right)^{2}+\left(y_{k, n e a r}-y_{k}^{a b s}\right)^{2}} \\
& R_{o b s}=\sqrt{\left(x_{k, n e a r}-x_{k}^{a b s}\right)^{2}+\left(y_{k, n e a r}-y_{k}^{a b s}\right)^{2}}+R_{\text {robot }}
\end{aligned}
$$

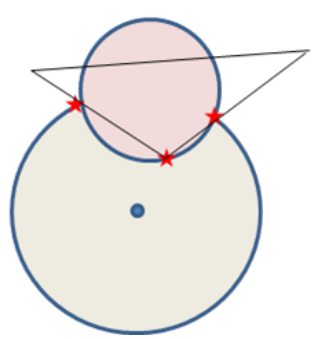

Fig. 1 Encirclement

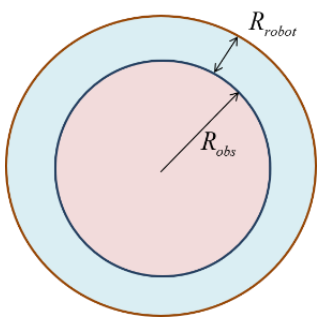

Fig. 2 Enlargement

During the process of obtaining a virtual obstacle, to make it sure that no part of an obstacle be missed in the LMS measurement, an appropriate sampling rate may be necessary, but these and other details of treatable shapes are not covered.

\section{B. Algorithm for Obstacle Avoidance}

The key idea of the algorithm is combining the resultant of the tangential vector from the encircled obstacle and the gravity vector to the target, to define the direction of the robot movement. The tangent vector is computed at the middle point or the nearest point among the three points obtained by the LMS. The gravity vector is computed at the robot position due to a point gravitational field centered at the target. The direction of the robot movement may be expressed as follows:

$$
\vec{D}_{\text {res }}=\overrightarrow{ \pm m}_{k, d i r}+\vec{F}_{\text {attr }}
$$

The magnitude of the tangential vector is taken proportional to the square of the radius of virtual obstacle:

$$
\operatorname{mag}\left(\vec{m}_{k, d i r}\right)=\eta \times R_{o b s}^{2}
$$




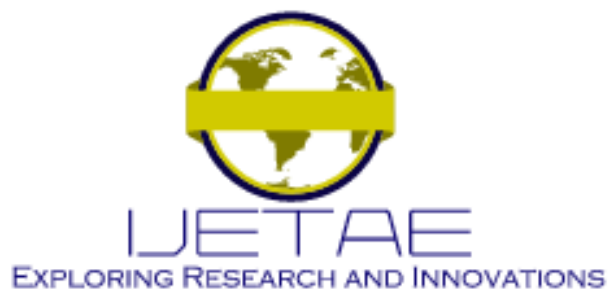

International Journal of Emerging Technology and Advanced Engineering

Website: www.ijetae.com (E-ISSN 2250-2459, Scopus Indexed, ISO 9001:2008 Certified Journal, Volume 11, Issue 10, October 2021)

where $\eta$ denotes a proportional constant, here taken as 4 and this value is selected by try and error.

To prevent zigzagging in the resulting robot movement, we separate two modes and each mode has two cases. Mode 1 is when at least one point which is obtained by LMS is not located on a line (Fig. 3). Case 1 and case 2 are defined in such a way to choose a shorter path to the target point. If the inner product of the previous (n-1)-st heading direction and the present $\mathrm{n}$-th tangential vector is positive, use the resultant vector as the next heading direction, and if negative, use the negative resultant vector as the next heading direction. That is,

$$
\begin{aligned}
& \vec{m}_{k, \operatorname{dir}(n)} \bullet \vec{D}_{r e s(n-1)}>0 \rightarrow \vec{D}_{r e s(n)}=+\vec{D}_{r e s(n-1)} \\
& \vec{m}_{k, \operatorname{dir}(n)} \bullet \vec{D}_{\text {res }(n-1)}<0 \rightarrow \vec{D}_{r e s(n)}=-\vec{D}_{r e s(n-1)}
\end{aligned}
$$

If all three points are located on one line, then mode 2 is applied on Fig. 4. In this case, the radius of the circle(obstacle) is infinite, making the magnitude of the tangential vector infinite; the gravity vector is then ignored. The obstacles are considered as a long wall and the mobile robot moves along the wall. In this mode,

$$
\begin{aligned}
& \vec{D}_{\text {res }}=\overrightarrow{ \pm m}_{k, d i r}+\vec{F}_{\text {attr }} \approx \overrightarrow{ \pm m}_{k, \text { dir }} \\
& \operatorname{mag}\left(\vec{m}_{k, d i r}\right)=\eta \times R_{o b s} \approx \infty
\end{aligned}
$$

Case 1 and 2 are applied in the same way as for mode 1 . Overall procedure of the developed method is shown as a flow chart in Fig. 6.

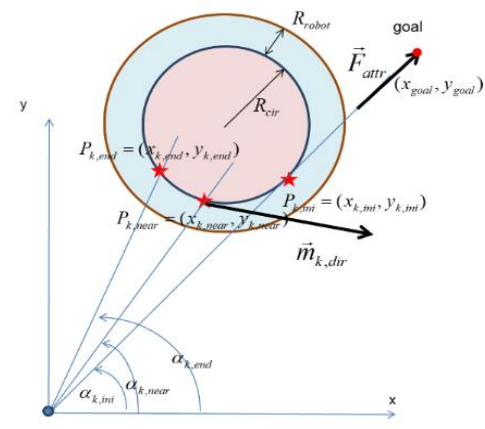

Fig. 3 Notations and applied vectors (Mode 1)

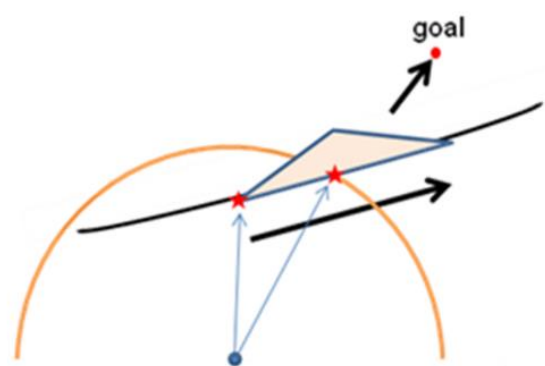

Fig. 4 Applied vectors (Mode 2)

\section{Local Path Optimization Algorithm}

The purpose of this paper is to propose methods of local path planning and emergency obstacle avoidance for Unmanned Ground Vehicles (UGVs). We proposed an optimal path generation algorithm to avoid obstacles in local environment, to generate the shortest path to goal, and to consider safety of UGVs. To propose an algorithm satisfying two conditions, we suggest a fuzzy controller that solves optimization equations by setting object functions and constraints and actively controls weights to make object function to adapt to arbitrary environments.

\section{Optimal Path Design}

To make a bulky GV to operate in narrow hallways, object functions and constraints are defined as shown below. In each time step, robot's next position $X_{n}$ is calculated from this optimization problem. The definitions of symbols used in this equation are shown in Table 2.

TABLE 2.

EQUATION VARIABLE DEFINITIONS

\begin{tabular}{|c|l|}
\hline Variables & \multicolumn{1}{|c|}{ Definition } \\
\hline$x_{\text {vehicle }}$ & Current vehicle position, $\mathrm{x}$-coordinate \\
\hline$y_{\text {vehicle }}$ & Current vehicle position, $\mathrm{y}$-coordinate \\
\hline$x_{\text {goal }}$ & Target position, $\mathrm{x}$-coordinate \\
\hline$y_{\text {goal }}$ & Target position, y-coordinate \\
\hline$\alpha, \beta$ & Weighting factors for the two objectives \\
\hline$\delta, \delta_{\max }$ & $\begin{array}{l}\text { Distance to the nearest point of an obstacle from } \\
\text { the next robot position, maximum safe distance }\end{array}$ \\
\hline$\gamma, \gamma_{\max }$ & Orientation angle, maximum orientation angle \\
\hline$V, V_{\max }$ & Vehicle speed, maximum speed \\
\hline $\mathrm{n}$ & The n-th time step \\
\hline $\mathrm{N}$ & The number of time steps to the target \\
\hline
\end{tabular}




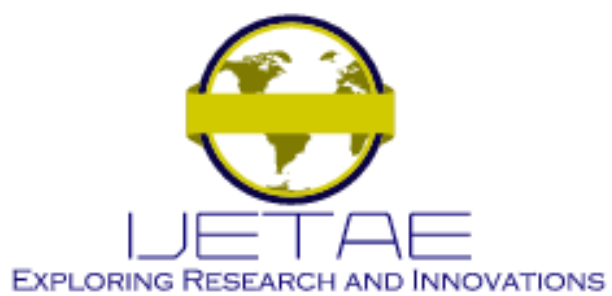

International Journal of Emerging Technology and Advanced Engineering

Website: www.ijetae.com (E-ISSN 2250-2459, Scopus Indexed, ISO 9001:2008 Certified Journal, Volume 11, Issue 10, October 2021)

$$
\begin{aligned}
& f=\min \left[J\left(X_{n}\right)\right] \\
& \text { subject to } \quad g_{i}\left(X_{n}\right) \leq 0, i=1,2 \\
& \text { and } \quad h\left(X_{n}\right)=0 \\
& \text { where } \quad X_{n}=\left[x_{\text {vehicle }}, y_{\text {vehicle }}\right]^{T} \\
& \qquad\left(X_{n}\right)=\Psi\left(\widetilde{\eta_{n}}\right)+\Omega\left(\widetilde{\zeta \eta_{n}}\right) \\
& \left.\Psi\left(\widetilde{\eta_{n}}\right)=\frac{1}{2} \widetilde{\eta_{n}^{T}} \alpha \widetilde{\zeta \eta_{n}}\right) \\
& \Omega\left(\widetilde{\zeta_{n}}\right)=\frac{1}{\left.\frac{1}{2} \widetilde{\eta_{n}^{T}} \beta \widetilde{\zeta_{n}}\right)} \\
& g_{1}=\delta_{\max }-\left|\delta_{n}\right| \\
& g_{2}=\left|\gamma_{n}\right|-\gamma_{\max } \\
& h=V_{n}-V_{\max }, \quad n=1, \ldots, N
\end{aligned}
$$

When UGVs operate, the most important factors are how fast and short the vehicle reaches the goal and securing safety distance to avoid collision with obstacles. These two factors are contradicting. If the vehicle keeps long distance from obstacles to avoid collisions, arrival time will increase. On the other hand, shortening arrival time and travel distance make the vehicle not to keep the safety distance. To control these two contradictory factors, we apply weights to object functions. To give steering constraint as shown in Eq. 16, we assumed UGVs as Ackermann vehicles to limit the maximum steering angle are and we assumed that the vehicle is running in constant speed of $V_{\text {max }}$. Also, we set the constraints to allow a UGV to maintain a minimum approach distance larger than a certain constant value to secure the safety from obstacles. The equations of object function and constraints are shown below.

$$
\begin{aligned}
\widetilde{\eta_{n}} & =\left|\left[x_{\text {goal }}, y_{\text {goai }}\right]^{T}-X_{n}\right| \\
\widetilde{\zeta_{n}} & =\left|\zeta_{\text {obs }(n)}-X_{n}\right|
\end{aligned}
$$

In the Eq.12, object function consists of two terms, and the first term $(\Psi)$ is to make UGVs to arrive at the goal as fast and short as possible (Eq. 18). The second term $(\Omega)$ is to run safely keeping a certain distant from obstacles (Eq. 19). Here, $\zeta_{o b s}$ is the absolute coordinate of the point where the closest obstacle is sensed. Eq. 15 is the minimum safety distance from UGV to obstacles, and $\delta_{n}$ is the distance from current vehicle position to the closest point on a sensed, which is set to $0.2 \mathrm{~m}$.
In addition, in Eq. 16, the maximum steering angle $\gamma_{\max }$ is set to 30 degrees applying the kinematic constraints. In Eq. 17, the vehicle speed $V_{\max }$ is assumed to be $1 \mathrm{~m} / \mathrm{s}$ and applied this to the simulation.

\section{Weight Control Method}

To make UGVs to reach the goal fast and safe avoiding obstacles in an arbitrary environment, we propose weight control technique using fuzzy controller. In the factors of the object function, Eq. 13 and 14, weight $\alpha$ and $\beta$ is set to control the importance. Eq. 13 is a function for arriving fast and short to the goal, and importance can be adjusted by selecting weight aproperly. In addition, Eq. 14 is a function for securing the distance from the vehicle to obstacles, and importance can be adjusted by selecting weight $\beta$ properly.

Therefore, membership function-based and rule setbased fuzzy controller is designed to control the weights of object function. We use the minimum distance from the vehicle to obstacles measured using LMS as a control input to control weight $\alpha$. The output of the fuzzy controller is weight $\alpha$, and $\beta$ is calculated using Eq. 20.

$$
\alpha+\beta=1
$$

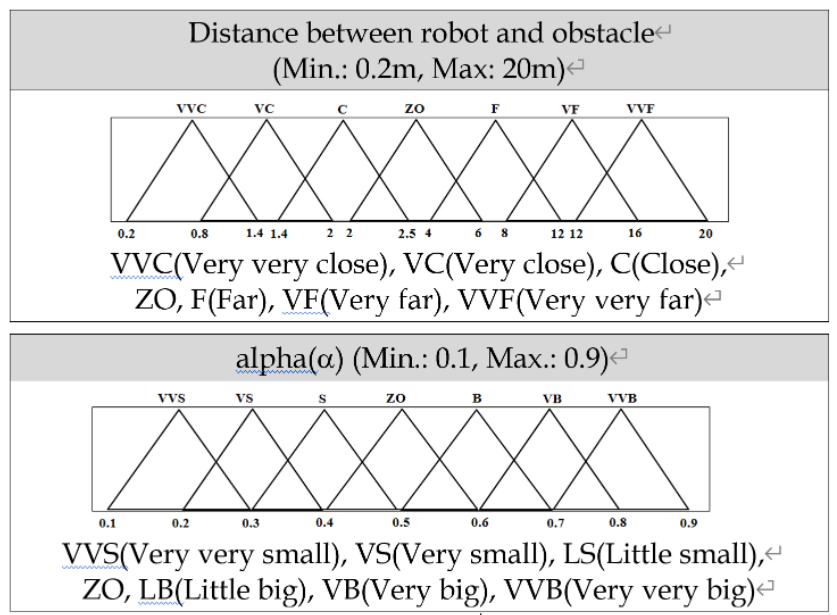

Fig. 5 Fuzzy input and output

Input and output variables are defined using seven membership functions, and it is shown in Fig. 5. The minimum and maximum values of the input are set to $0.2 \mathrm{~m}$ and $20 \mathrm{~m}$. This is because of the constraint that keeps the vehicle minimum $0.2 \mathrm{~m}$ away from obstacles and the maximum range of LMS. 


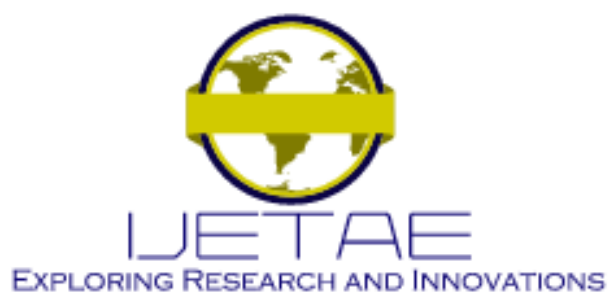

International Journal of Emerging Technology and Advanced Engineering

Website: www.ijetae.com (E-ISSN 2250-2459, Scopus Indexed, ISO 9001:2008 Certified Journal, Volume 11, Issue 10, October 2021)

Also, the range of output value $\alpha$ is from ' 0 ' to ' 1 ' as shown in Eq. 20. The process selecting the rule set is very important when designing fuzzy controllers. Therefore, it is better to be selected by an experienced personnel or expert. To set the proper rules for UGV running in complex environment, the vehicle must move away from the obstacles when it approaches to obstacle which is to decrease weight $\alpha$, so we selected 7 rules as shown in Table 3.

TABLE 3.

FUZZY LOGIC OUTPUT

\begin{tabular}{|c|c|}
\hline Selected 7 rules & Output (speed) \\
\hline If input A is VVC & VVS \\
\hline If input A is VC & VS \\
\hline If input A is C & LS \\
\hline If input A is ZO & ZO \\
\hline If input A is F & LB \\
\hline If input A is VF & VB \\
\hline If input A is VVF & VVB \\
\hline
\end{tabular}

\section{Simulation Result}

\section{A. Algorithm for Obstacle Avoidance}

Simulation results of a typical example by VFF and proposed method are compared to see some of the characteristics. Fig.7 shows those from VFF algorithm and Fig. 8 from the proposed algorithm. The sampling frequency used for both the simulation was 0.01 seconds. A visual examination of the path of the present algorithm indicates smoother and less curvy than the other. The time of completion of the motion to the target was 5 seconds and 3 seconds for the VFF and our algorithm, respectively.

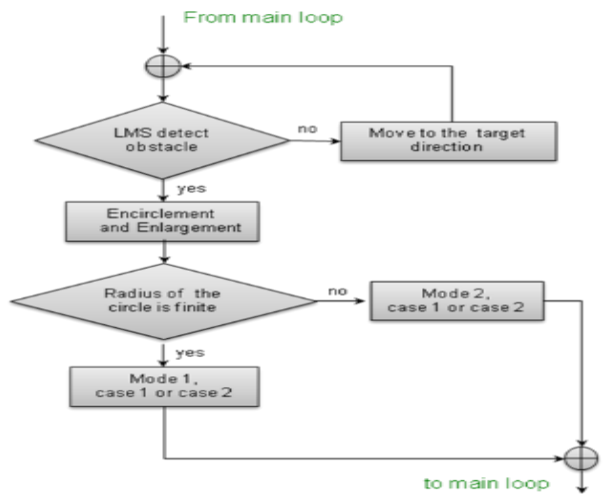

Fig. 6 Flow chart of proposed method

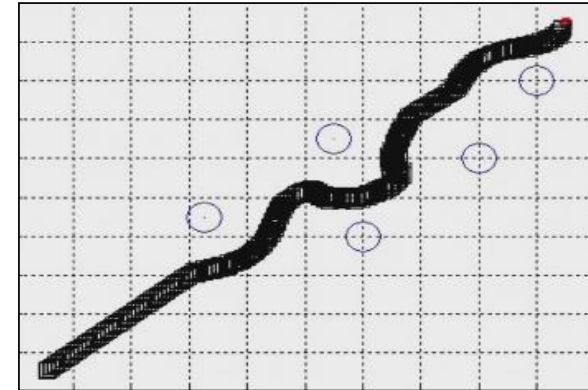

Fig. 7 Previous algorithm (VFF)

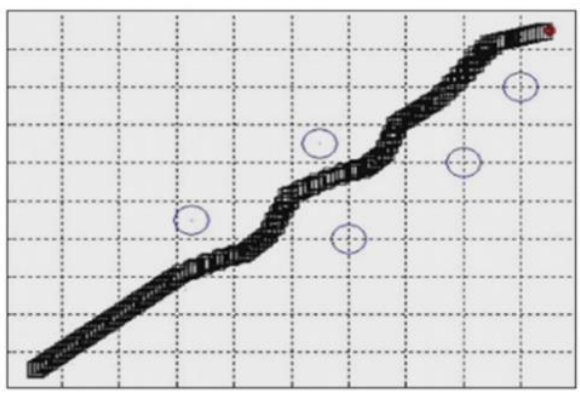

Fig. 8 Proposed algorithm

\section{B. Verifying the Performance of Proposed Method}

The method is demonstrated using several cases to verify performances: multiple obstacles, a long wall type obstacle, and a U-shaped obstacle. In the drawings, rectangles are the original obstacles and yellow circles are the real time virtual obstacles. They may not be seen on print.

Fig. 9 shows good performance for a typical case of several obstacles. Fig. 10 and Fig. 11 demonstrate that the method has overcome the local minimum problems in local path planning that many other algorithms have not.

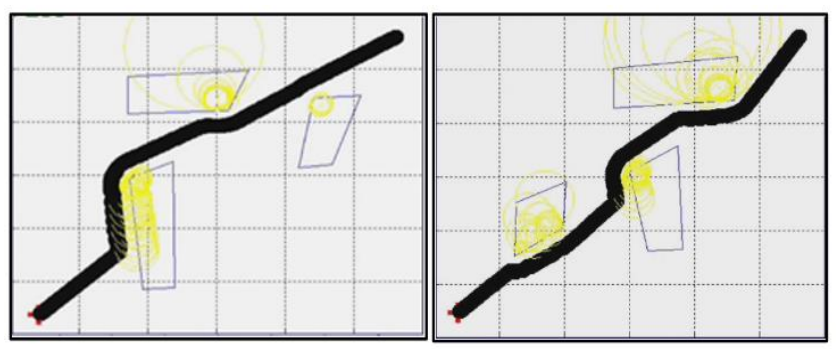

Fig. 9 Multiple obstacles 


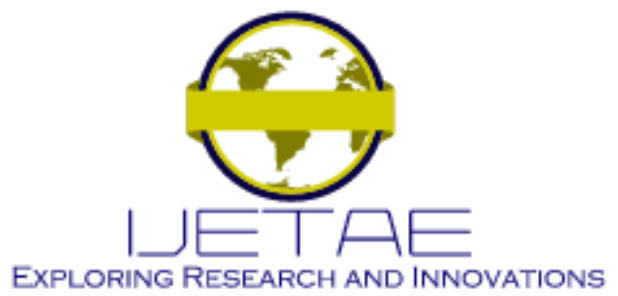

International Journal of Emerging Technology and Advanced Engineering

Website: www.ijetae.com (E-ISSN 2250-2459, Scopus Indexed, ISO 9001:2008 Certified Journal, Volume 11, Issue 10, October 2021)

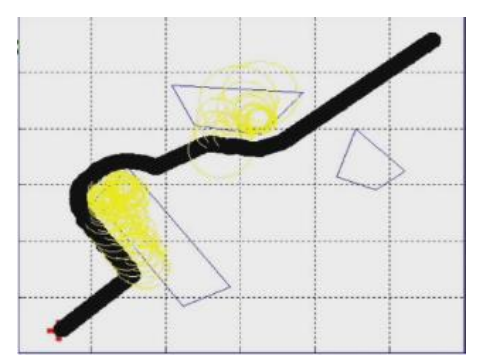

Fig. 10 Long wall type obstacle

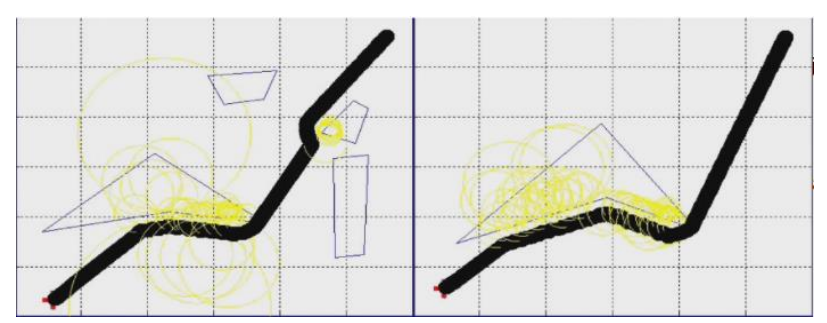

Fig. 11 U-shaped obstacle

\section{Simulation Results (LOCAl OPTIMIZATION)}

In order to verify the proposed algorithm, we constructed the similar vehicle, sensor model and used them in the arbitrary simulation environments in Matlab. First, the weight control and optimization algorithm are compared with the algorithm which utilized the fixed weight values. The width of the corridor is $5 \mathrm{~m}$ which is less than twice width of the SUGV. The first environment consists of single path with frequent 90 degree turning, and the second environment is constructed with squares arranged as chess board. There are several possible paths for the robot in the second environment unlike the first one. The performance and the effectiveness of the weight control algorithm can be seen in the second experimental results as the robot moves well in such a complex surrounding. Fig. 12 shows the overall system architecture of the simulation.

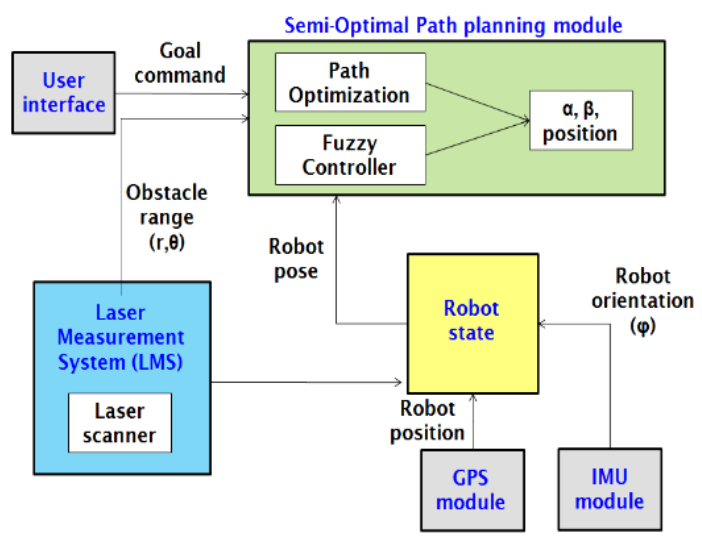

Fig. 12. overall system architecture
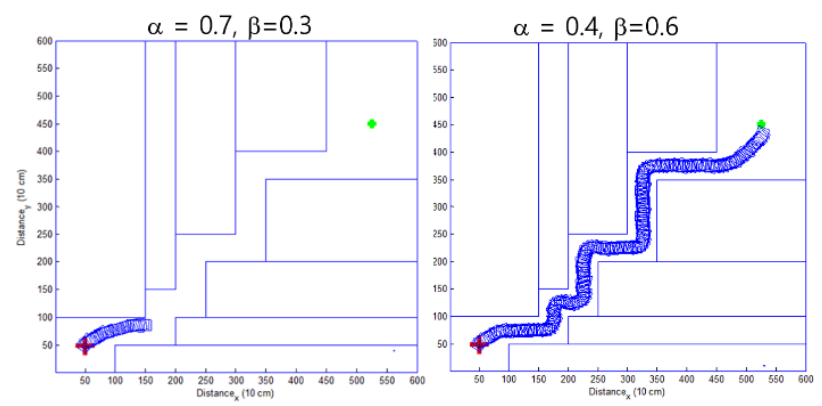

Fig. 13 Fixed weight model (environment 1)
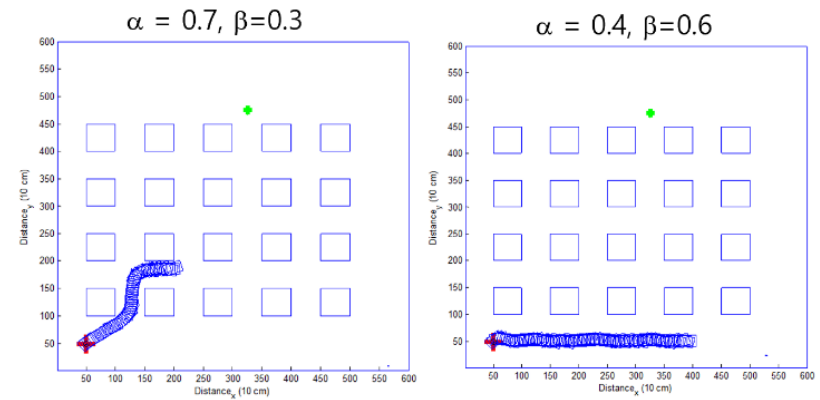

Fig. 14. Fixed weight model (environment 2) 


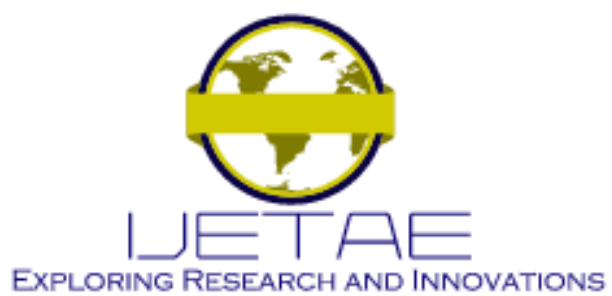

International Journal of Emerging Technology and Advanced Engineering

Website: www.ijetae.com (E-ISSN 2250-2459, Scopus Indexed, ISO 9001:2008 Certified Journal, Volume 11, Issue 10, October 2021)

\section{A. Fixed weight algorithm}

Fixed weight algorithm utilizes the fixed weight value, $\alpha$ and $\beta$, of the cost function without controlling from Fuzzy controller. Fig 13 and 14 show the result of this algorithm. $\alpha$ and $\beta$ are fixed as $\{0.7,0.3\},\{0.4,0.6\}$ respectively at each first and second simulation. The goal position of the robot is upper right corner in the graph.

In the first simulation, the algorithm is failed by the collision to the wall when the weight value is $\{0.7,0.3\}$. This suggests that the user made a wrong choice of deciding the weight values in this environment. The vehicle collided to the wall since the $\alpha$, which indicates the effect of selecting the shortest path from current to goal location, is high, and $\beta$, which indicates the effect of moving as far as from the obstacles, is low. Therefore, the algorithm was successful with the weight values $\{0.4,0.6\}$ since the robot tries more to put some distance from obstacles than to select the shortest path from current to goal location.

On the other hand, in the second simulation, both weight values which are applied in the first simulation are unsuccessful. The simulation using first weight values is failed due to the same reason in the first simulation, and the second simulation using second weigh values is also failed since the robot cannot pass between obstacles due to high $\beta$ value. As a result, the robot moves the outside of the obstacles and can't get into the obstacle to get to the goal position (Fig. 14).

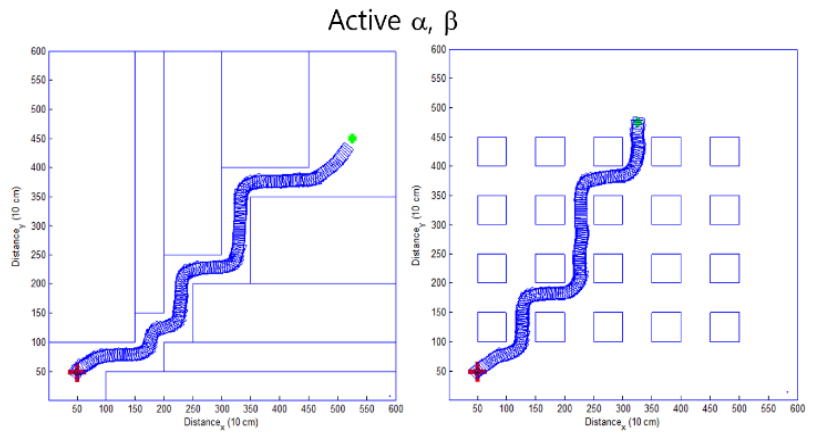

Fig. 15 Varying weight model

\section{B. Varying weight algorithm}

The varying weight algorithm actively controls the weight values, $\alpha$ and $\beta$, with the consideration of environment using the Fuzzy controller.
This algorithm can be applied in arbitrary environments since it controls weight values by continuously checking the distance of obstacles and goal position. The algorithm is also tested in the same environments used with fixed weight algorithm. The results are shown in Fig. 15, and it is successful in both environments.

Both fixed and varying weight algorithm satisfy the constraints, once the robot arrives at the goal position in the simulation. However, the fixed weight algorithm was not able to drive on the shortest path, which is optimal, with many other successful weight values. On the other hand, the varying weight algorithm successfully generated the optimized path by changing the weight values in term of local environment. Especially, in the second simulation, the robot was able to safely arrive to the goal with little time period.

\section{Experimental results}

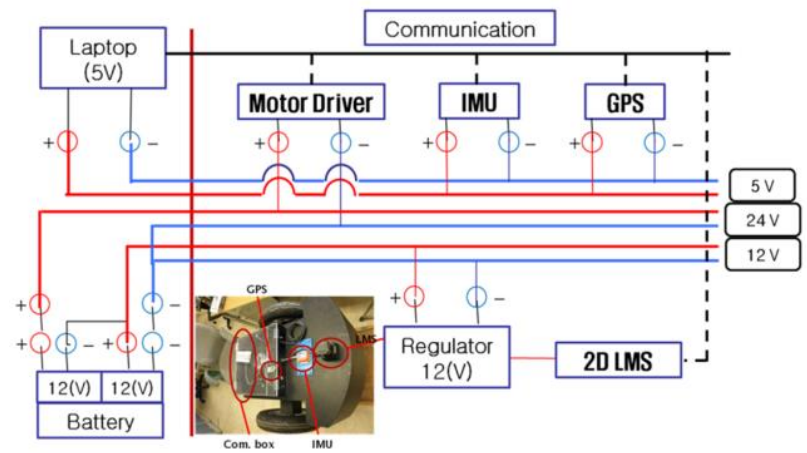

Fig. 16 Specification of the mobile robot.

On the real test we use two wheeled mobile robot and the specifications are shown in Fig. 16. LMS and IMU sensors are used to implement and they are same as sensors which will be attached to final platform.

Fig. 17 presents that the UGV is driving well on the corridor like environment without oscillation and messy area. When performing implementation, we consider sampling time as 100 millisecond for updating wheel speed command. The main problem was weight parameter tuning, so we tuned the weight parameters to find semi-optimal value during several test. Although there are no animations, we found out that the robot is wondering well without collisions. Later, on the final demonstration, we are going to use platform to show UGV reaching to the goal using odometry (encoder data). 


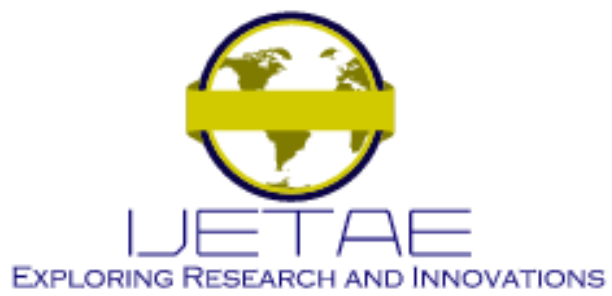

International Journal of Emerging Technology and Advanced Engineering Website: www.ijetae.com (E-ISSN 2250-2459, Scopus Indexed, ISO 9001:2008 Certified Journal, Volume 11, Issue 10, October 2021)
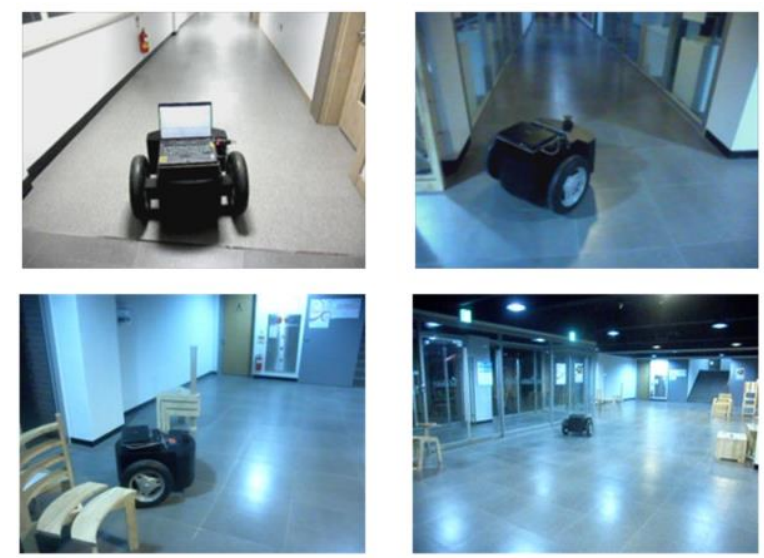

Fig. 17 Corridor like environment (upper figure), messy environment (lower figure)

\section{CONCLUSION}

In this paper, finally we proposed VTV and Local Optimal path algorithm that the robot can generate the optimal path to the goal position without colliding obstacles as adapting Fuzzy controller which continuously optimizes the weight values of the cost function in terms of local environment. The algorithm was verified using simulation in Matlab, and showed the good performance as keeping distance between the robot and obstacles more than $0.2 \mathrm{~m}$ at every state of the robot. This indicates that the vehicle's motion satisfies the constraint at all time, and the algorithm successfully controls the weight values from Fuzzy controller. For the future work, the algorithm should be tested in real corridor and complex environment and verified experimentally.

\section{REFERENCES}

[1] J. Borenstein, "Real-time obstacle avoidance for fast mobile robots", IEEE Transactions on Systems, Man, and Cybernetics, Vol. 19, No. 5, Sept./Oct. 1989, pp. 1179-1187.
[2] J. Borenstein, Y. Koren, "the vector field histogram-fast obstacle avoidance for mobile robots", IEEE Journal of Robotics and Automation, Vol.7, No.3, June 1991, pp. 278-288.

[3] Dieter Foxy, Wolfram Bardy, Sebastian Thrunyz, "The Dynamic Window Approach to Collision Avoidance", August 1995, IAI-TR95-13

[4] J. Minguez, "Nearness Diagram (ND) Navigation: Collision Avoidance in Troublesome Scenarios", IEEE Transactions on Robotics and Automation, vol. 20, no. 1, 2004, pp. 45-59.

[5] Kyung Woon Kwak, Hae Kwan Jeong, Soo Hyun Kim and Yoon Keun Kwak, "VTV: Real Time Obstacle Avoidance of Mobile Robots for Local Path Planning using LMS", International Conference on Control, Automation and Systems, Seoul, Korea, 2008, pp. 1597-1600.

[6] http://www.globalsecurity.org/military/systems/ground/fcs.htm

[7] http://www.globalsecurity.org/military/systems/ground/fcs-ugv.htm

[8] http://en.wikipedia.org/wiki/PackBot

[9] http://foster-miller.qinetiq-na.com/lemming.htm

[10] Bradski,G. R. "Computer Vision face tracking for use in a perceptual interface", Intel Technology Journal, 2dn Quarter,1998.

[11] Cheng, Y, "Mean Shift, mode seeking, and clustering", IEEE Transaction on Pattten Analysis and Machine Intelligence, vol 17, pp. 790-799, 1999.

[12] Dorin Comaniciu, Peter Meer, "Mean Shift: A Robust Approach Toward Feature Space Analysis", IEEE Transaction on Pattern Analysis and Machine Intelligence, vol. 24, No. 5, pp. 603-619, 2002.

[13] Intel Corportation (2001): OpenSource Computer Vision Library Reference Manual, 123456-001

[14] G. R. Bradski, "Computer vision face tracking for use in a perceptual user interface”, Interface, vol. 2, no. 2, 1998.

[15] J. Allen and R. Xu, "Object tracking using camshift algorithm and multiple quantized feature spaces", Proceedings of the Pan-Sydney area, vol. 36, pp. 3-7, 2004.

[16] P. Fieguth and D. Terzopoulos, "Color-based tracking of heads and other mobile objects at video frame rates", Proceedings of IEEE Computer Society Conference on Computer Vision and Pattern Recognition, pp. 21-27, 1997.

[17] O. dris Nouar, G. Ali, and C. Raphaël, "Improved object tracking with camshift algorithm", pp. 657-660, 2006. 